\title{
Can Elderly Patients with Peritoneal Metastasis Induced by Appendiceal or Colorectal Tumours Benefit from Cytoreductive Surgery (CRS) and Hyperthermic Intraperitoneal Chemotherapy (HIPEC)?
}

This article was published in the following Dove Press journal: Clinical Interventions in Aging

\author{
Sicheng Zhou',* \\ Qiang Feng ${ }^{1} * *$ \\ Jing Zhang ${ }^{2}$ \\ Haitao Zhou' \\ Zheng Jiang' \\ Jianwei Liang' \\ Wei Pei ${ }^{1}$ \\ Qian Liu' \\ Zhixiang Zhou' \\ Xishan Wang' \\ 'Department of Colorectal Surgery, \\ National Cancer Center/National Clinical \\ Research Center for Cancer/Cancer \\ Hospital, Chinese Academy of Medical \\ Sciences and Peking Union Medical \\ College, Beijing, I0002I, People's \\ Republic of China; ${ }^{2}$ Department of \\ Abdominal Surgery, Huanxing Cancer \\ Hospital, Beijing, 100I22, People's \\ Republic of China
}

*These authors contributed equally to this work
Correspondence: Jianwei Liang; Wei Pei Email Liangjw1976@I63.com; peiweifbwk@163.com
Purpose: Cytoreductive surgery (CRS) added with hyperthermic intraperitoneal chemotherapy (HIPEC) can improve the survival rate of certain patients with peritoneal metastasis (PM). However, the perioperative safety and long-term survival of this intricate and possibly life-threatening procedure in elderly patients ( $\geq 65$ years) remain controversial.

Methods: Patients with PM due to appendiceal or colorectal tumours who underwent CRS/ HIPEC were evaluated systematically at the National Cancer Center of China and the Huanxing Cancer Hospital between June 2017 and June 2019. The recruited subjects were retrospectively categorized into elderly (age $\geq 65$ ) and non-elderly (age $<65$ ) groups according to their age. Clinical and pathological features, postoperative outcomes, and prognoses were gathered and analysed.

Results: Both groups had similar overall morbidity $(56.0 \%$ vs $38.7 \%, P=0.130)$ and grade $3 /$ 4 morbidity $(28.0 \%$ vs $20.0 \%, P=0.403)$ after CRS/HIPEC. However, more patients in the elderly group suffered from ileus postoperatively ( $16.0 \%$ vs $2.6 \%, P=0.033)$. After a followup period of a median of 20 months, it was concluded that elderly patients had significantly worse 3 -year overall survival (OS) than non-elderly patients ( $16.3 \%$ vs $51.4 \%, P=0.001)$. Independent prognostic factors were identified to be a high peritoneal carcinomatosis index (PCI) score (HR, 1.10, 95\% CI, 1.04-1.16; $P=0.001)$ and age $\geq 65$ (HR, 2.42, 95\% CI, $1.32-4.45 ; P=0.004)$ were independent prognostic factors through cox regression analysis.

Conclusion: CRS and HIPEC are related with an elevated prevalence of postoperative ileus but not with the overall morbidity or the grade $3 / 4$ morbidity in elderly patients. However, since worse survival outcomes were observed more commonly in elderly patients compared to younger patients from CRS+HIPEC, this complex and potentially life-threatening procedure should be considered carefully in patients aged $\geq 65$ years.

Keywords: elderly patients, CRS, HIPEC, morbidity, survival

\section{Introduction}

Ageing is a recognized predisposing factor for colorectal cancer. ${ }^{1}$ With the extension of life expectancy, the population of elderly patients with colorectal cancer is increasing. ${ }^{2}$ Metastasis to the peritoneum is the second commonest type of colorectal cancer (CRC) metastasis, it has been reported that $8 \% \sim 15 \%$ of CRC patients have already had developed peritoneal metastasis (PM) at the time of diagnosis. ${ }^{3}$ 
The 5-year survival of patients with PM induced by CRC is only $20 \% \sim 25 \%$, the median survival time is only $6 \sim 9$ months, and the 1-year survival rate of patients with malignant ascites is less than $10 \%{ }^{4,5}$ The standard treatment approach has become hyperthermic intraperitoneal chemotherapy (HIPEC) added with cytoreductive surgery (CRS), which have been reported to improve the prognosis considerably. ${ }^{6-9}$

The principle of the CRS+HIPEC procedure is to achieve complete cytoreduction ( $\mathrm{CC} 0 / 1)$ by extensive excision of the tumour lesions visible in the peritoneum, abdomen and pelvis. Then, chemotherapy medications will be introduced to infuse the tumour bed to remove the residual microlesions. Therefore, this complex procedure has received much attention, mostly concerning patient morbidity and mortality due to its invasive nature. ${ }^{10-12}$ Furthermore, for most elderly patients with underlying diseases, the incidence of cardiovascular and respiratory complications during the perioperative period is relatively high. The development of major postoperative complications will lead to poor prognosis and even death. ${ }^{13,14}$ Therefore, a single-centre retrospective analysis procedure was designed to examine the perioperative safety and long-term survival of CRS+HIPEC in elderly patients (age $\geq 65$ years) with PM from appendiceal or colorectal cancer.

\section{Patients and Methods}

\section{Patient Inclusion}

One hundred patients who underwent CRS and HIPEC for peritoneally disseminated colorectal or appendiceal malignancies between June 2017 and June 2019 in the Huanxing Cancer Hospital were included in the study. The standards for selecting patients are as follows: (1) patients who have pathologically diagnosed appendiceal or colorectal cancer; (2) who are aged between 18 and 75 years; and (3) who has an Eastern Cooperative Group (ECOG) score of less than or equal to 1. (4) patients who had received three complete courses of HIPEC procedure. Patients with the following history were excluded (1) a past history of other malignancies; (2) who are receiving palliative care, for instance, ostomy or bypass surgeries; (3) neutrophil count of the peripheral blood $<2000 \times 10^{9} / \mathrm{L}$ or platelet count $<100 \times 10^{9} / \mathrm{L}$; (4) unusual function of livers indicated by a serum total bilirubin (TBIL) level $>21 \mu \mathrm{mol} / \mathrm{L}$ or alanine transaminase (ALT) level $>40 \mathrm{U} / \mathrm{L}$; and (5) unusual kidney function marked by a serum creatinine level
$>106 \mu \mathrm{mol} / \mathrm{L}$ or urea level $>7.1 \mathrm{mmol} / \mathrm{L}$. In the end, 100 qualifying patients were recruited in this study, who all gave informed consent and the procedure complies with the Declaration of Helsinki. The ethics committee of the Chinese Academy of Medical Sciences Cancer hospital (NCC 2017-YZ-026, Oct 17, 2017) approved this research.

\section{Preoperative Management}

Patients received regular preoperative assessments in order to evaluate their general health condition in order to quantify the peritoneal carcinomatosis index (PCI) and to assess any metastasis; these evaluations included lab exams, abdominal CT scans, and pelvic MRI. The multidisciplinary teams (MDTs) reviewed all possible CRS +HIPEC cases, along with radiologists, medical and surgical oncologists to formulate a complete treatment plan with consensus. Data on patient demographics and perioperative indicators were prospectively collected and analysed from an institutional database. Chemotherapy toxicity indices including liver, kidney, and blood toxicities, neutrophil and platelet counts; TBIL, ALT, urea and creatinine levels were obtained in the morning of postoperative days (PODs) 1, 3, and 5. Patients whose liver and kidney were severely impaired or were experiencing myelosuppression were withdrawn from further HIPEC procedure and excluded from the research. Sixty-five was used as the age cut-off value, and all patients were categorised retrospectively into elderly (age $\geq 65$ ) and nonelderly (age $<65)$ groups according to their age.

\section{Operative Details}

After performing a complete adhesiolysis and examination of the abdomen, the degree of PM was determined by the PCI score ranging from 0 to 3 , grading each of the 13 identified areas within the abdomen. ${ }^{15,16}$ Based on the different locations of PM, CRS would indicate different surgeries and peritonectomy procedures ranging from pelvic and anterior peritonectomy, omentectomy, ovariectomy, to hysterectomy, all adhering to the Sugarbaker technique. ${ }^{17}$ To assess residual lesions, the completeness of the cytoreduction score (CC score) was documented after each operation. CC- $0 / 1$ is considered to be complete cytoreduction (CC-0 meaning no perceptible pathology; CC-1 meaning nodules less than $0.25 \mathrm{~cm}$ ), with $\mathrm{CC}-2 / 3$ cases considered incomplete cytoreduction. ${ }^{18}$ Incomplete cytoreduction means there is residual nodule that can be seen with naked eyes, indicating the presence of residual tumour. Patients receiving palliative care such as bypass 
surgery or ostomy were excluded. In the end, 15 patients with resectable liver metastases were identified and underwent surgeries for the removal of liver metastases after CRS.

A closed technique for HIPEC was administered after cytoreduction and fashioning of the intestinal anastomoses. Intraperitoneal chemotherapy used Raltitrexed $\left(4 \mathrm{mg} / \mathrm{m}^{2}\right)$ and Oxaliplatin $\left(200 \mathrm{mg} / \mathrm{m}^{2}\right)$ on their own or combined with lobaplatin $\left(50 \mathrm{mg} / \mathrm{m}^{2}\right)$. Following the placement of catheters, patients received a mixture of chemotherapy agents and 3 $\mathrm{L}$ of saline into the abdominal and pelvic cavities for $60 \mathrm{~min}$ at $43^{\circ} \mathrm{C}$, followed by two more HIPEC procedures with same chemotherapeutic combinations for the same length of perfusion time, and four catheters stay in their original positions; this was carried out on the 2nd and 4th days after surgery while in the ward by two surgical specialists who have over 20 years of experience in the field of digestive system surgery.

\section{Postoperative Morbidity and Mortality}

Using the Common Terminology Criteria for Adverse Events (CTCAE) classification, any postoperative complication was documented and evaluated within 30 days. ${ }^{19}$ The function of livers was assessed quantitatively through serum ALT, and function of kidneys was checked by levels of serum creatinine. Toxicity indices (liver, kidney, and blood), including neutrophil and platelet counts, ALT, and creatinine levels were recorded on postoperative days (PODs) 1, 3, and 5 in the morning.

\section{Follow-Up}

Most of the PM patients underwent postoperative adjuvant chemotherapy formulated by two consultant oncologists at their own discretion. Every patient needed to attend follow-up appointments either face-to-face or over telephones every trimonthly for the first two years and then every 6-12 monthly for the next 3 years until patient deceased caused by recurrence and metastases of tumours or until July 31, 2020. At each follow-up visit, abdominal and pelvic CT scans were taken, along with tumour markers. The long endpoint for the current study was 3-year overall survival (OS).

\section{Statistical Processing}

Statistical analysis of the gathered data using computers was performed using the SPSS 24.0 for Windows (IBM Corp, Armonk, NY, USA). Quantitative data were presented as the mean \pm SD and evaluated with for independent abnormal and normal distributions using the Mann-Whitney U-tests and Student's t-tests, respectively. Categorical data were presented as percentages, and the test groups were compared using $\chi^{2}$ tests or Fisher's exact tests as demanded. The Kaplan-Meier method was adopted for precise calculation of the survival analysis, and data were processed with the log-rank test. The multivariate Cox regression model was utilised to appraise all significant univariate variables for their independent prognostic value. Statistical significance level was asset at the $P$ value of less than 0.05 .

\section{Results}

\section{Patients' Traits and Surgical Outcomes}

Patient demographics and clinical features are displayed in Table 1. In total, 100 subjects were recruited in the study, their mean age was $56.5 \pm 11.5$ years. Based on the patient's age at the time of diagnosis, 25 (25.0\%) were assigned to the elderly group (age $\geq 65$ ), and $75(75.0 \%$ ) were allocated to the non-elderly group (age <65). The frequency of cardio-pulmonary comorbidities was significantly higher in the elderly group $(52.0 \%$ vs $20.0 \%$, $P=0.002$ ), resulting in a higher ASA score. The most prevalent primary tumour in both the non-elderly $(n=64$, $85.3 \%)$ and elderly groups $(n=22,93.0 \%)$ was colorectal malignant tumour $(P=0.836)$. The mean PCI score was similar between the elderly group and the non-elderly group (10.6 vs $11.2, P=0.673$ ), and most patients achieved complete cytoreduction in both groups $(60.0 \%$ vs $72.0 \%$, $P=0.261)$. No significant difference regarding gender, BMI, PM presentation, primary tumour, preoperative CEA and CA19-9 levels, ascites, and liver metastasis were observed $(P>0.05)$.

Table 2 displays intra- and postoperative outcomes, and similar type of operation and HIPEC regimen were administered to both groups. The average operating duration was $235.6 \mathrm{~min}$ in the elderly group and $254.3 \mathrm{~min}$ in the non-elderly group $(P=0.237)$. The mean estimated blood loss was basically equal between the two groups (127.6 mL vs $122.8 \mathrm{~mL}, P=0.857$ ). As for postoperative recovery, it is worth noting that the first flatus time of patients in the elderly group was considerably longer (5.4 days vs 3.1 days, $P=0.008$ ). No meaningful differences were seen regarding the time taken to recover to a regular diet or the postoperative hospital stay.

Postoperative complications are described in Table 2. Elderly patients had elevated overall morbidity rate; however, this was not statistically significant $(56.0 \%$ vs $38.7 \%$, 
Table I Clinicopathological Characteristics of 100 Patients Underwent CRS/HIPEC

\begin{tabular}{|c|c|c|c|c|}
\hline Characteristics & Overall $(n=100)$ & Age $\geq 65$ Years $(n=25)$ & Age $<65$ Years $(n=75)$ & $\boldsymbol{P}$ \\
\hline Age, year (mean $\pm S D)$ & $56.5 \pm 11.5$ & $68.9 \pm 3.5$ & $50.6 \pm 9.6$ & $<0.00$ \\
\hline Gender & & & & 0.908 \\
\hline Male & $53(53.0)$ & $13(52.0)$ & $40(53.3)$ & \\
\hline Female & $47(47.0)$ & $12(48.0)$ & $35(46.7)$ & \\
\hline $\mathrm{BMI}, \mathrm{kg} / \mathrm{m}^{2}($ mean $\pm \mathrm{SD})$ & $23.0 \pm 2.7$ & $23.5 \pm 2.5$ & $22.7 \pm 2.8$ & 0.376 \\
\hline ASA score & & & & 0.039 \\
\hline 1 & $38(38.0)$ & $6(24.0)$ & $32(42.7)$ & \\
\hline II & $58(58.0)$ & $16(64.0)$ & $42(56.0)$ & \\
\hline III & $4(4.0)$ & $3(12.0)$ & I (I.3) & \\
\hline Cardio-pulmonary comorbidities & & & & 0.002 \\
\hline Presence & $28(28.0)$ & $13(52.0)$ & $15(20.0)$ & \\
\hline Absence & $72(72.0)$ & $12(48.0)$ & $60(80.0)$ & \\
\hline Presentation of PM & & & & 0.193 \\
\hline Synchronous & $6 I(6 I .0)$ & I8 (72.0) & $43(57.3)$ & \\
\hline Metachronous & $39(39.0)$ & $7(28.0)$ & $32(42.7)$ & \\
\hline Primary tumour & & & & 0.836 \\
\hline Colon & $63(63.0)$ & $17(68.0)$ & $46(61.3)$ & \\
\hline Rectum & $23(26.0)$ & $5(25.0)$ & $18(24.0)$ & \\
\hline Appendix & $14(14.0)$ & $3(12.0)$ & II (I4.7) & \\
\hline Preoperative CEA level, $\mathrm{ng} / \mathrm{mL}($ mean $\pm \mathrm{SD})$ & $30.1 \pm 61.8$ & $31.5 \pm 76.9$ & $29.7 \pm 59.3$ & 0.927 \\
\hline Preoperative CAI9-9 level, ng/mL (mean \pm SD) & $63.4 \pm 84.0$ & $68.4 \pm 105.2$ & $56.4 \pm 65.9$ & 0.655 \\
\hline Histology & & & & 0.465 \\
\hline Adenocarcinoma & $66(66.0)$ & $15(60.0)$ & $51(68.0)$ & \\
\hline Mucinous/signet-ring & $34(34.0)$ & $10(40.0)$ & $24(32.0)$ & \\
\hline $\mathrm{PCl}$ sorce (mean $\pm \mathrm{SD})$ & $11.0 \pm 5.8$ & $10.6 \pm 5.0$ & $11.2 \pm 6.1$ & 0.673 \\
\hline Liver metastases & & & & 0.506 \\
\hline Presence & $14(14.0)$ & $5(25.0)$ & $9(12.0)$ & \\
\hline Absence & $86(86.0)$ & $20(75.0)$ & $66(88.0)$ & \\
\hline Ascites & & & & 0.557 \\
\hline Presence & $4 I(4 I .0)$ & $9(36.0)$ & $32(42.7)$ & \\
\hline Absence & $59(59.0)$ & $16(64.0)$ & $43(57.3)$ & \\
\hline CC score & & & & 0.901 \\
\hline CC 0-I & $69(69.0)$ & $17(68.0)$ & $52(69.3)$ & \\
\hline CC 2-3 & 31 (3I.0) & $8(32.0)$ & $23(20.7)$ & \\
\hline
\end{tabular}

Abbreviations: BMI, body mass index; ASA, American Society of Anesthesiologists; PM, peritoneal metastasis; PCl, peritoneal carcinomatosis index; CC score, cytoreduction score.

$P=0.130$ ). The overall Clavien-Dindo grade $3 / 4$ morbidity was $22.0 \%$, in which $28.0 \%$ were from the elderly group and $20.0 \%$ from the non-elderly group $(P=0.403)$. However, patients in the elderly group suffered more frequently from ileus postoperatively than the other group ( $16.0 \%$ vs $2.6 \%, P=0.033$ ). The commonest grade $3 / 4$ complications after CRS-HIPEC were abscesses within the pelvic cavity $(6.0 \%)$, ileus $(6.0 \%)$, followed by wound infections (3.0\%), anastomosis leakage (3.0\%) and intra-abdominal haemorrhage $(3.0 \%)$. Four patients in total $(4.0 \%)$ required reoperation as a result of postoperative complications $(P=1.000)$, out of which one patient was in the elderly group (4.0\%) and three patients in the non-elderly group $(4.0 \%)$, the reoperation was due 
Table 2 Perioperative Data of 100 Patients Underwent CRS/HIPEC

\begin{tabular}{|c|c|c|c|c|}
\hline Characteristics & Overall $(n=100)$ & Age $\geq 65$ Years $(n=25)$ & Age $<65$ Years $(n=75)$ & $\boldsymbol{P}$ \\
\hline Operation method & & & & 1.000 \\
\hline Laparoscopic surgery & $18(18.0)$ & $4(16.0)$ & $14(18.7)$ & \\
\hline Open surgery & $82(82.0)$ & $21(84.0)$ & $61(81.3)$ & \\
\hline HIPEC regimen & & & & 0.204 \\
\hline Lobaplatin+Oxaliplatin+Raltitrexed & $48(48.0)$ & $10(40.0)$ & $4 \mathrm{I}(54.7)$ & \\
\hline Oxaliplatin+Raltitrexed & $52(52.0)$ & $15(60.0)$ & $34(45.3)$ & \\
\hline Operative time, $\min (\operatorname{mean} \pm S D)$ & $240.6 \pm 69.5$ & $235.6 \pm 66.5$ & $254.3 \pm 70.8$ & 0.237 \\
\hline Estimated blood loss, $\mathrm{mL}$ (mean $\pm \mathrm{SD}$ ) & $124.0 \pm 113.5$ & $127.6 \pm 122.0$ & $122.8 \pm 112.1$ & 0.857 \\
\hline Postoperative complications & $43(43.0)$ & $14(56.0)$ & $29(38.7)$ & 0.130 \\
\hline Postoperative complications (grades III, IV) & $22(22.0)$ & $7(28.0)$ & $15(20.0)$ & 0.403 \\
\hline Arrhythmia & I (I.0) & I (4.0) & $0(0)$ & 0.250 \\
\hline Pneumonia & $2(2.0)$ & I (4.0) & $\mathrm{I}(\mathrm{I} .3)$ & 0.439 \\
\hline Pleural effusion & $2(2.0)$ & I (4.0) & $\mathrm{I}(\mathrm{I} .3)$ & 0.439 \\
\hline Anastomotic leakage & $3(3.0)$ & I (4.0) & $2(2.6)$ & 1.000 \\
\hline Ileus & $6(6.0)$ & $4(16.0)$ & $2(2.6)$ & 0.033 \\
\hline Gastrointestinal hemorrhage & $2(2.0)$ & $0(0)$ & $2(2.6)$ & 1.000 \\
\hline Renal failure & $\mathrm{I}(\mathrm{I} .0)$ & I (4.0) & $0(0)$ & 0.250 \\
\hline Urinary retention & $2(2.0)$ & I (4.0) & $0(0)$ & 0.250 \\
\hline Abdominal abscess & $6(6.0)$ & I (4.0) & $5(6.7)$ & 1.000 \\
\hline Rectovaginal fistula & $\mathrm{I}(\mathrm{I} .0)$ & $0(0)$ & $\mathrm{I}(\mathrm{I} .3)$ & 1.000 \\
\hline Intra-abdominal hemorrhage & $3(3.0)$ & $0(0)$ & $3(4.0)$ & 0.571 \\
\hline Wound infection & $3(3.0)$ & $0(0)$ & $3(4.0)$ & 0.571 \\
\hline Time to first flatus, day (mean \pm SD) & $3.5 \pm 0.8$ & $5.4 \pm 0.9$ & $3.1 \pm 0.8$ & 0.008 \\
\hline Time to regular diet, day (mean $\pm S D)$ & $5.8 \pm 3.4$ & $7.2 \pm 3.6$ & $5.5 \pm 3.3$ & 0.122 \\
\hline Postoperative hospital stay, day (mean \pm SD) & $15.3 \pm 5.9$ & $19.2 \pm 7.4$ & $14.8 \pm 5.1$ & 0.018 \\
\hline Re-operation & $4(4.0)$ & I (4.0) & $3(4.0)$ & 1.000 \\
\hline Mortality & $0(0)$ & $0(0)$ & $0(0)$ & 1.000 \\
\hline
\end{tabular}

Abbreviation: HIPEC, hyperthermic intraperitoneal chemotherapy.

to extensive abscesses of pelvic cavities, postoperative haemorrhage, and perforations of small bowels caused by ileus. There were no deaths in the two groups during the 30-day hospitalization.

Statistically significant discrepancies between the two groups relating to chemotherapy toxicity indices were not observed, including neutrophil count, platelet, creatinine, and ALT levels on PODs 1, 3, and $5(P>0.05)$ (Figures 1-4).

\section{Patients' Mortalities}

The median duration of follow-ups was 20 (ranging between 3 and 40) months, the median survival for patients was 25 months, and the projected 1-, 2- and 3 -year OS rates were $76.9 \%, 53.2 \%$, and $41.2 \%$, respectively (Figure 5). Elderly patients had 1-, 3-, and 5 -year OS rates of $51.4 \%, 27.2 \%$, and $16.3 \%$, and nonelderly patients had 1-, 3-, and 5-year OS rates of $84.0 \%$, $62.0 \%$, and 51.4\% (Figure 6). The Kaplan-Meier curves showed that elderly patients had a much better OS rate than those in the younger group ( $P=0.001)$.

Using the Cos univariate regression analysis, it was possible to identify age at operation, PCI and CC scores, and overall postoperative morbidities as predisposing factors of OS (Table 3). Independent prognostic factors were concluded through the multivariable Cox regression analysis, to be PCI score and age at the time of operation. Patients aged $\geq 65$ (HR, 2.42, 95\% CI, 1.32-4.45; $P=0.004$ ) and those with high PCI scores (HR, 1.10, 95\% CI, 1.04-1.16; $P=0.001)$ had considerably poorer overall survival (Table 3 ). 


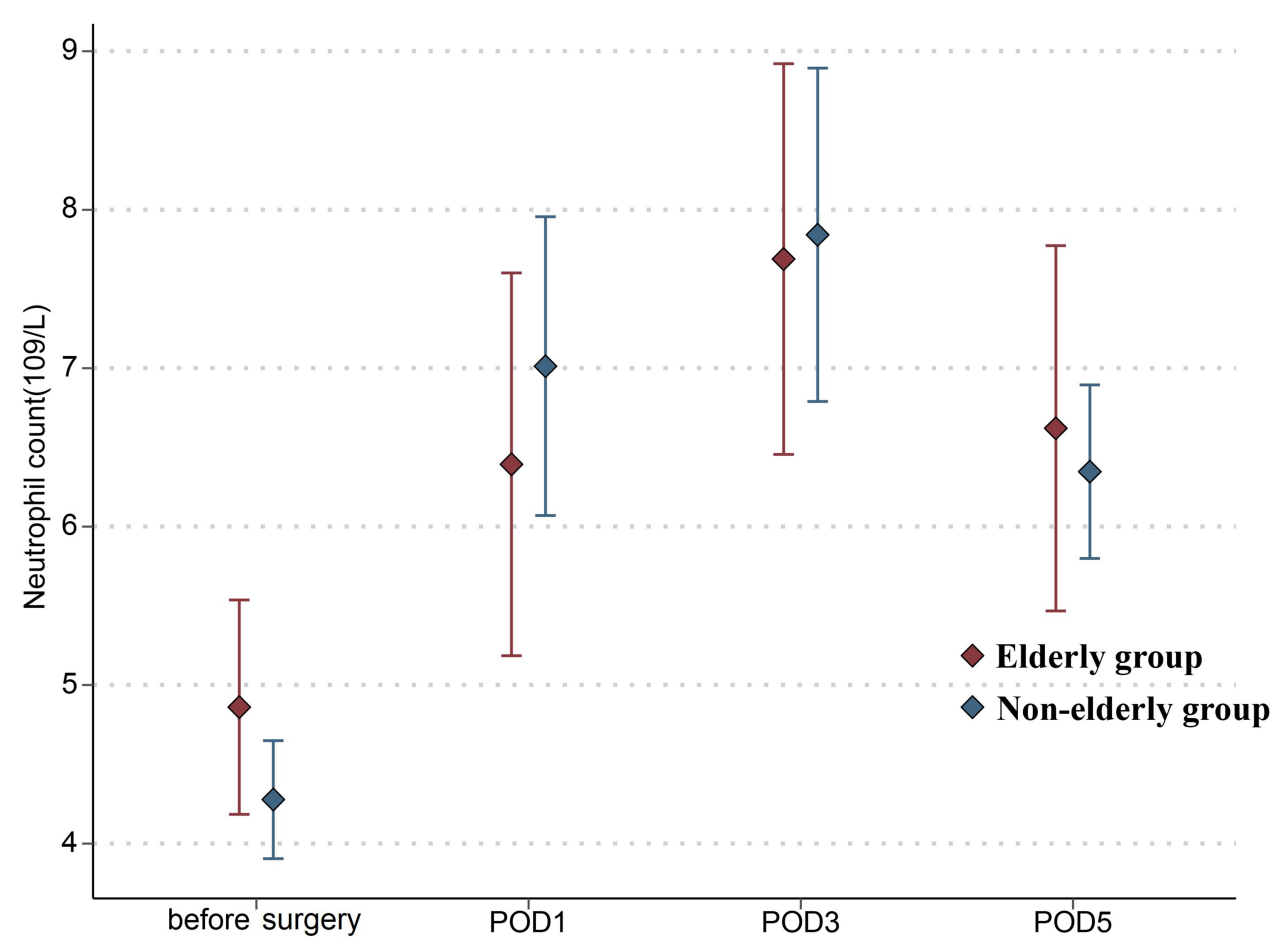

Figure I Changes in the neutrophil count in elderly and non-elderly groups on days I, 3, and 5 after surgery.

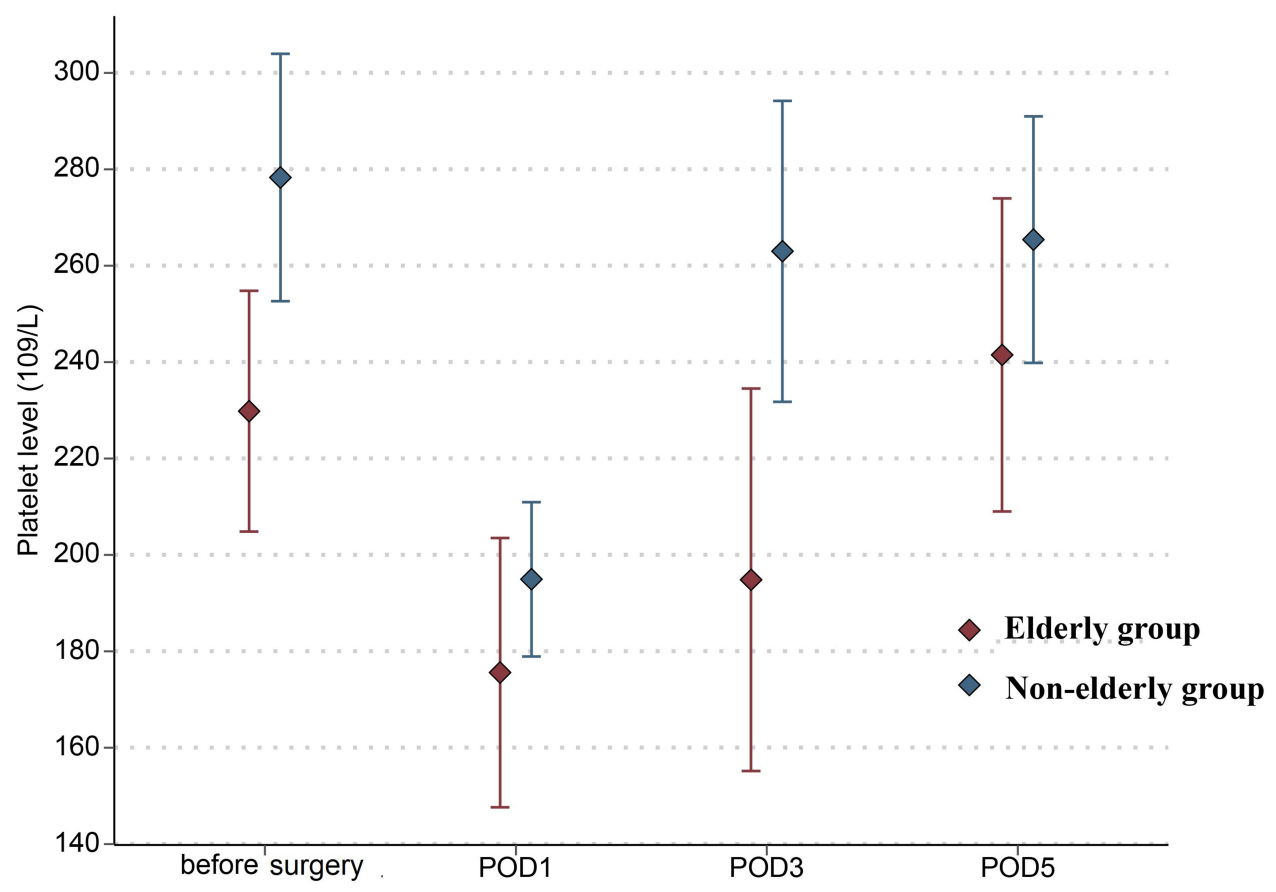

Figure 2 Changes in the platelet level in elderly and non-elderly groups on days I, 3, and 5 after surgery.

\section{Discussion}

Results of this present study demonstrated that in elderly patients (age $\geq 65$ years) with colorectal cancer peritoneal metastases, CRS plus HIPEC could be performed safely with acceptable overall morbidity and grade $3 / 4$ morbidity.
However, it should be noted that elderly patients were more likely to develop postoperative ileus. Although patients underwent this complex and potentially life-threatening procedure that required both visceral and peritoneal resection to achieve complete cytoreduction, no death occurred within 30 


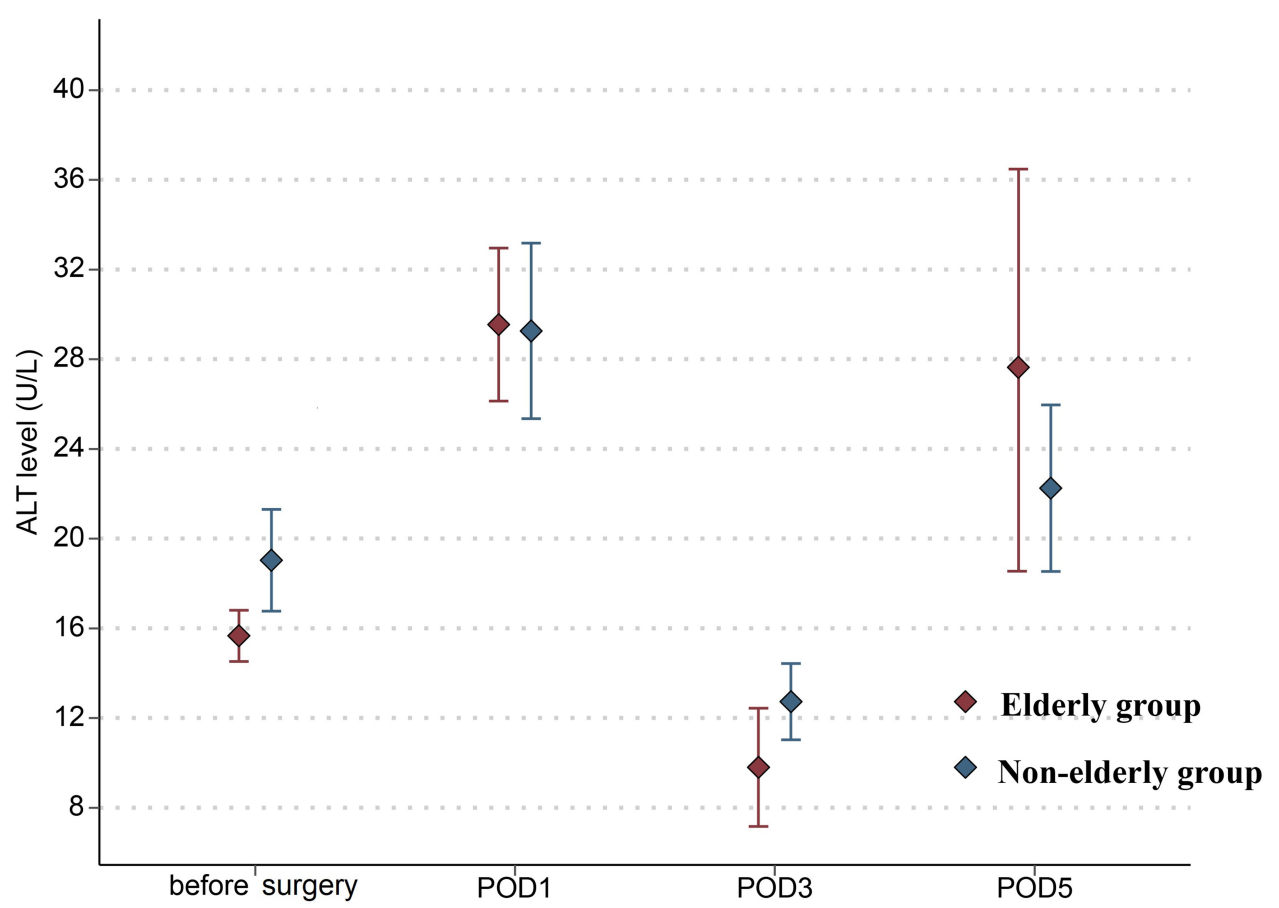

Figure 3 Changes in the ALT level in elderly and non-elderly groups on days I, 3, and 5 after surgery.

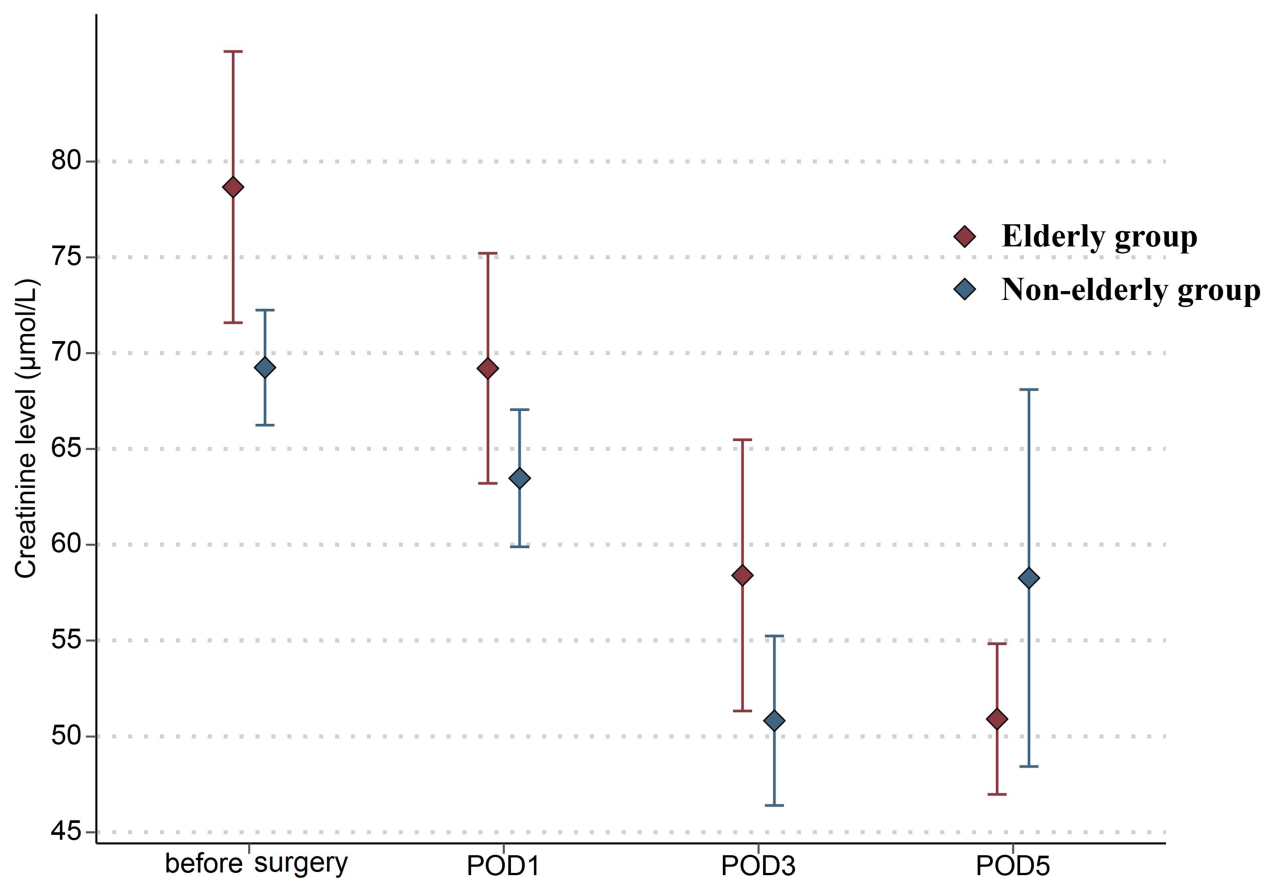

Figure 4 Changes in the creatinine level in elderly and non-elderly groups on days I, 3, and 5 after surgery.

days during the postoperative period. In terms of long-term prognosis, our findings propose that elderly patients with peritoneal metastases induced by colorectal tumours do not profit from CRS/HIPEC.
The associated morbidity and mortality of the CRS/ HIPEC procedure are receiving increasing attention, especially for elderly individuals with more complicated underlying diseases and low surgical tolerance. 


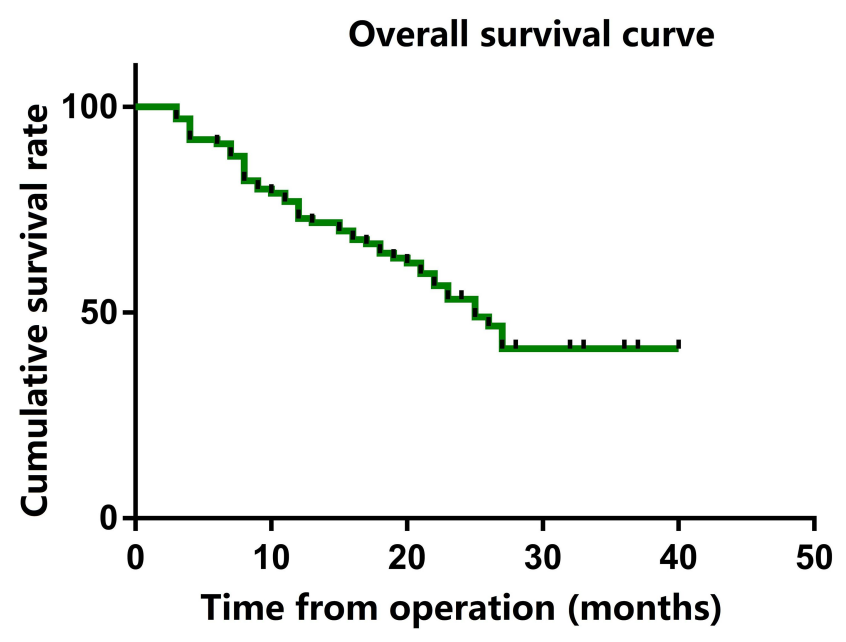

Figure 5 Overall survival curve in entire group.

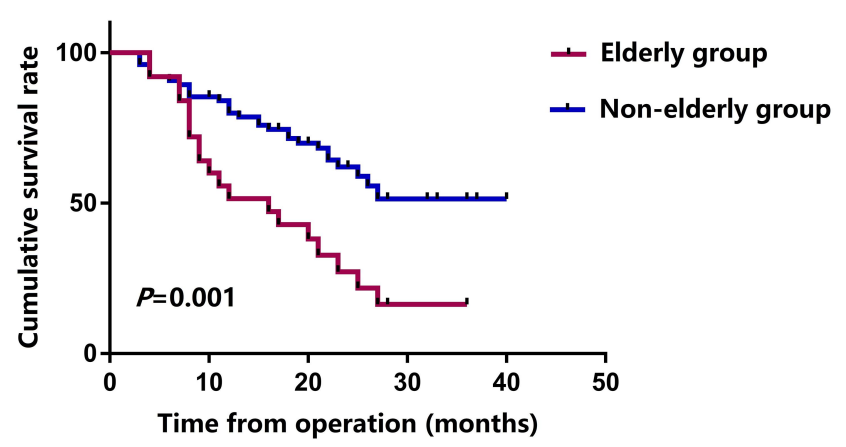

Figure 6 Overall survival curve in two groups.

Wong et al stated that compared to non-elderly patients, the incidence of high-grade complications did not increase significantly after CRS/HIPEC (44.4\% vs $24.5 \%, P=0.79)$ among elderly patients. ${ }^{20}$ Hence, the study suggested that age should not be a contraindication of CRS/HIPEC. Beckert et al also provided strong evidence that elderly patients experienced more overall postoperative complications $(76.0 \%$ vs $47.0, P=0.048)$; however, there was no notable increase in grade 3-4 complications (21.0 vs $12.0 \%$, $P=1.000) .{ }^{21}$ In the present study, the PCI score and operation time between both groups of patients were well balanced, suggesting a high degree of similarity in the extent of surgery, which is consistent with the above literature. Although higher incidence of cardiopulmonary comorbidities was observed in elderly patients, consequently the preoperative ASA score was also elevated, the present study proved that elderly patients and non-elderly patients had similar grade $3 / 4$ morbidity ( 28.0 vs $20.0 \%, P=0.403$ ), and the toxicity of chemotherapy was not significantly increased. A high incidence of overall morbidity in the elderly group was also seen, however this was not statistically significant ( $56.0 \%$ vs $38.7 \%, P=0.130)$. Furthermore, in terms of grade 3-4 complications, a significantly elevated proportion of postoperative ileus in elderly patients (16.0 vs $2.6 \%, P=0.033)$ was found, which may be due to the extensive distribution of chemotherapy drugs in the abdomen, the inhibitory effect of chemotherapy drugs on gastrointestinal motility and the slow recovery of gastrointestinal function after intestinal anastomosis in elderly patients.

The overall survival of elderly patients with peritoneal metastases after CRS/HIPEC still remains controversial. Wong et al suggested that older adults could achieve similar 5 -year OS (51.0\% vs 59.6\%, $P=0.88)$ and disease-free survival (DFS) $(23.3 \%$ vs $53.3 \%, P=0.60)$ rates as younger individuals after $\mathrm{CRS}+\mathrm{HIPEC} .^{20}$ By contrast, a meta-analysis consisting of ten research papers stated detailed data on elderly patients after CRS+HIPEC and showed a consistent lower survival rate for elderly patients who underwent CRS +HIPEC across all research settings and procedures compared with non-elderly patients. ${ }^{22}$ Our study surprisingly found that the 3-year OS rate of elderly patients after CRS/ HIPEC was considerably poorer than that of younger patients ( $16.3 \%$ vs $51.4 \%, P=0.001)$. It was also indicated by the multivariable Cox regression analysis that age $\geq 65$ years was another independent prognostic factor (HR, 2.42, 95\% CI, $1.32-4.45 ; P=0.004)$. The decreased survival rate after CRS/HIPEC in elderly patients with colorectal cancer and concurrent peritoneal metastases may be due to the following reasons. (1) Most elderly patients had not completed the adequate systemic intravenous chemotherapy due to physical or psychological reasons after the CRS/HIPEC procedure. (2) Numerically, the incidence of overall morbidity in elderly patients increased by approximately $20 \%$ in the present study, although this was not statistically significant. The manifestation of postoperative complications may degrade patients' general conditions and thus interfere with subsequent adjuvant therapies or treatment for limiting recurrence. (3) The quality of life of elderly patients after receiving this difficult and potentially life-threatening operation is obviously affected, which may easily lead to tumour recurrence and progression. A series of studies aimed at describing longitudinal quality-of-life in elderly patients after CRS/ HIPEC are necessary.

The most significant limitation of our investigation is that it was a retrospective cohort study with a limited 
Table 3 Univariate and Multivariate Cox Regression Analysis of Overall Survival in 100 Patients After CRS/HIPEC

\begin{tabular}{|c|c|c|c|c|}
\hline \multirow[t]{3}{*}{ Variables } & \multicolumn{4}{|c|}{ Overall Survival } \\
\hline & \multicolumn{2}{|c|}{ Univariate Analysis } & \multicolumn{2}{|c|}{ Multivariate Analysis } \\
\hline & HR (95\% CI) & $\mathbf{P}$ & HR (95\% Cl) & $\mathbf{P}$ \\
\hline Gender: female/male & $\mathrm{I} .43(0.8 \mathrm{I}-2.52)$ & 0.217 & & \\
\hline Age at operation ( $<65$ years $/ \geq 65$ years) & $2.57(I .43-4.60)$ & 0.002 & $2.42(1.32-4.45)$ & 0.004 \\
\hline Preoperative chemotherapy (no/yes) & $\mathrm{I} .05(0.54-2.0 \mathrm{I})$ & 0.894 & & \\
\hline Synchronous/metachronous & $0.99(0.56-1.76)$ & 0.966 & & \\
\hline Site of original (Colorectum/Appendix) & $0.41(0.13-1.32)$ & 0.135 & & \\
\hline Histology (adenocarcinoma/mucinous) & $1.13(0.64-2.00)$ & 0.672 & & \\
\hline Preoperative CEA level & $1.01(1.00-1.01)$ & 0.076 & & \\
\hline Preoperative CA I9-9 level & $1.00(0.99-1.01)$ & 0.202 & & \\
\hline Liver metastases (no/yes) & $1.54(0.74-3.18)$ & 0.245 & & \\
\hline HIPEC regimen (lobaplatin/non-lobaplatin) & $1.42(0.79-2.53)$ & 0.298 & & \\
\hline Presence of ascites (no/yes) & $1.32(0.75-2.33)$ & 0.335 & & \\
\hline $\mathrm{PCl}$ score & $1.11(1.06-1.16)$ & $<0.001$ & $1.10(1.04-1.16)$ & 0.001 \\
\hline CC score $(0-1 / 2-3)$ & $3.26(I .83-5.81)$ & $<0.001$ & $1.76(0.93-3.32)$ & 0.083 \\
\hline Overall postoperative complications (no/yes) & $2.55(1.40-4.62)$ & 0.002 & $1.43(0.76-2.68)$ & 0.267 \\
\hline Grade 3-4 Postoperative complication (no/yes) & I.5 I $(0.82-2.80)$ & 0.184 & & \\
\hline Neutropenia (no/yes) & $0.87(0.34-2.21)$ & 0.770 & & \\
\hline Thrombocytopenia (no/yes) & $0.98(0.39-2.48)$ & 0.964 & & \\
\hline
\end{tabular}

Abbreviations: HIPEC, hyperthermic intraperitoneal chemotherapy; PCl, peritoneal carcinomatosis index; CC score, cytoreduction score.

study sample of 100 patients, increasing the possibility of being biased. However, the multi-disciplinary team that specialises in colorectal cancer in our institution were in charge of treatment planning, and any data gathered were unabridged and reliable. Secondly, the elderly group had a higher percentage of patients with liver metastasis (25\% vs $12 \%$ ) and mucinous/signet ring cell carcinoma (40\% vs $32 \%)$, and these confounders could confuse the results. However, we have incorporated these factors into the multivariate Cox regression analysis to minimize the influence of confounding factors on the results. Therefore, large-scale randomized controlled studies are worth carrying out to verify our results.

In conclusion, it is safe to perform CRS/HIPEC in elderly patients, and the morbidity and mortality rates are acceptable. However, since elderly patients appear to obtain worse survival outcomes than younger patients from CRS+HIPEC, this complex and potentially lifethreatening procedure should be considered carefully in patients aged $\geq 65$ years.

\section{Ethical Statement}

This study was carried out in line with the local ethical guidelines and was permitted by the ethics committee of the Cancer Hospital, Chinese Academy of Medical Sciences (NCC 2017-YZ-026, Oct 17, 2017).

\section{Acknowledgments}

This research is funded by the Capital's Funds for Health Improvement and Research (2016-2-4022) and a prospective randomized controlled clinical trial of intraoperative intraperitoneal chemotherapy with raltitrexed for the prevention of peritoneal metastases from colorectal cancer in high risk patients (2020-044-KF).

\section{Disclosure}

The authors report no conflicts of interest or financial relations in this work.

\section{References}

1. Etzioni DA, Beart RW Jr, Madoff RD, et al. Impact of the aging population on the demand for colorectal procedures. Dis Colon Rectum. 2009;52(4):583-590. doi:10.1007/DCR.0b013e3181a1d183

2. Rasool S, Kadla SA, Rasool V, et al. A comparative overview of general risk factors associated with the incidence of colorectal cancer. Tumour Biol. 2013;34(5):2469-2476. doi:10.1007/s13277013-0876-y

3. Teo M. Peritoneal-based malignancies and their treatment. Ann Acad Med Singapore. 2010;39(1):54-57.

4. van Gestel YR, de Hingh IH, van Herk-sukel MP, et al. Patterns of metachronous metastases after curative treatment of colorectal cancer. Cancer Epidemiol. 2014;38(4):448-454. doi:10.1016/j.canep.2014.04. 004 
5. Mo S, Cai G. Multidisciplinary treatment for colorectal peritoneal metastases: review of the literature. Gastroenterol Res Pract. 2016;2016:1516259. doi:10.1155/2016/1516259

6. Bushati M, Rovers KP, Sommariva A, et al. The current practice of cytoreductive surgery and HIPEC for colorectal peritoneal metastases: results of a worldwide web-based survey of the Peritoneal Surface Oncology Group International (PSOGI). Eur J Surg Oncol. 2018;44(12):1942-1948. doi:10.1016/j.ejso.2018.07.003

7. Cashin PH, Mahteme H, Spång N, et al. Cytoreductive surgery and intraperitoneal chemotherapy versus systemic chemotherapy for colorectal peritoneal metastases: a randomised trial. Eur $J$ Cancer. 2016;53:155-162. doi:10.1016/j.ejca.2015.09.017

8. Van Driel WJ, Koole SN, Sikorska K, et al. Hyperthermic intraperitoneal chemotherapy in ovarian cancer. $N$ Engl J Med. 2018;378 (3):230-240. doi:10.1056/NEJMoa1708618

9. Klaver CEL, Wisselink DD, Punt CJA, et al. Adjuvant hyperthermic intraperitoneal chemotherapy in patients with locally advanced colon cancer (COLOPEC): a multicentre, open-label, randomised trial. Lancet Gastroenterol Hepatol. 2019;4(10):761-770. doi:10.1016/ S2468-1253(19)30239-0

10. Chua TC, Yan TD, Saxena A, Morris DL. Should the treatment of peritoneal carcinomatosis by cytoreductive surgery and hyperthermic intraperitoneal chemotherapy still be regarded as a highly morbid procedure? A systematic review of morbidity and mortality. Ann Surg. 2009;249(6):900-907. doi:10.1097/SLA.0b01 3e3181a45d86

11. Tan JW, Tan GHC, Ng WY, et al. High-grade complication is associated with poor overall survival after cytoreductive surgery and hyperthermic intraperitoneal chemotherapy. Int J Clin Oncol. 2020;25(5):984-994. doi:10.1007/s10147-01901609-5

12. Narasimhan V, Tan S, Kong J, et al. Prognostic factors influencing survival in patients undergoing cytoreductive surgery with hyperthermic intraperitoneal chemotherapy for isolated colorectal peritoneal metastases: a systematic review and meta-analysis.Colorectal Dis. 2020;22(11):1482-1495.

13. van Eeghen EE, den Boer FC, Loffeld RJ. Thirty days post-operative mortality after surgery for colorectal cancer: a descriptive study. J Gastrointest Oncol. 2015;6(6):613-617. doi:10.3978/j.issn.20786891.2015 .079
14. Arnarson Ö, Butt-Tuna S, Syk I. Postoperative complications following colonic resection for cancer are associated with impaired long-term survival. Colorectal Dis. 2019;21(7):805-815. doi:10.11 11/codi. 14613

15. Jacquet P, Sugarbaker PH. Clinical research methodologies in diagnosis and staging of patients with peritoneal carcinomatosis. Cancer Treat Res. 1996;82:359-374.

16. Elias D, Blot F, El Otmany A, et al. Curative treatment of peritoneal carcinomatosis arising from colorectal cancer by complete resection and intraperitoneal chemotherapy. Cancer. 2001;92(1):71-76. doi:10.1002/ 1097-0142(20010701)92:1<71::AID-CNCR1293>3.0.CO;2-9

17. Sugarbaker PH. Peritonectomy procedures. Ann Surg. 1995;221 (1):29-42. doi:10.1097/00000658-199501000-00004

18. Koh JL, Yan TD, Glenn D, et al. Evaluation of preoperative computed tomography in estimating peritoneal cancer index in colorectal peritoneal carcinomatosis. Ann Surg Oncol. 2009;16(2):327-333. doi:10.1245/s10434-008-0234-2

19. Hamashige S, Arquilla ER. Common terminology criteria for adverse events v3.0 (CTCAE). Principles Pract Clin Trial Med. 2008;42 (4):461-533.

20. Wong EYT, Tan GHC, Chia CSL, et al. Morbidity and mortality of elderly patients following cytoreductive surgery and Hyperthermic Intraperitoneal Chemotherapy (HIPEC). Asia Pac J Clin Oncol. 2018;14(2):e193-e202. doi:10.1111/ajco.12723

21. Beckert S, Struller F, Horvath P, et al. Overall morbidity but not mortality is increased in elderly patients following cytoreductive surgery and HIPEC. Langenbecks Arch Surg. 2015;400(6):693-698. doi:10.1007/s00423-015-1325-8

22. López-López V, Cascales-Campos PA, Schneider MA, et al. Cytoreductive surgery and hyperthermic intraperitoneal chemotherapy (HIPEC) in elderly patients. Syst Literature Rev Surg Oncol. 2016;25(4):378-384.
Clinical Interventions in Aging

\section{Publish your work in this journal}

Clinical Interventions in Aging is an international, peer-reviewed journal focusing on evidence-based reports on the value or lack thereof of treatments intended to prevent or delay the onset of maladaptive correlates of aging in human beings. This journal is indexed on PubMed Central, MedLine, CAS, Scopus and the Elsevier

\section{Dovepress}

Bibliographic databases. The manuscript management system is completely online and includes a very quick and fair peer-review system, which is all easy to use. Visit http://www.dovepress.com/ testimonials.php to read real quotes from published authors. 